\title{
Anthraquinones and Phenols as Intermediates in the Formation of Dark-Colored, Humic Acid-Like Pigments by Eurotium echinulatum ${ }^{1}$
}

\author{
C. Saiz-Jimienez, K. Haider and J. P. Martin²
}

\begin{abstract}
Eurotium echinulatum, a fungus isolated from a vertisol in southern Spain, formed humic acid-like pigments during growth in glucose-asparagine or gltreose- $\mathrm{NaNO}_{3}$ media. After 6 to 7 days the phenols, orsellinic; $p$-hydroxycinnamic, and $p$-hydroxybenzoic acids, and the anthraquinones, endocrocin, emodin, and physcion were detected in the culture media. Upon further development of the mycelial mats additional aromatic compounds were produced and after 4 to 6 weeks more than 50 different ether-extractable phenols and anthraquinones were detected. With further incubation the amounts and number of these compounds decreased and pigment formation in the media increased. After 2 to 3 months the culture media were dark brown to black and about 1 to $3.5 \mathrm{~g} / \mathrm{liter}$ of polymer could be recovered upon acidification of the culture medium. Sodium amalgam reduction of this polymer yielded numerous phenols, anthraquinones, anthrones, and possibly anthracene derivatives. Treatment with sodium dithionite yielded largely anthraquinones. The polymer contained 1 to $4.5 \% \mathrm{~N}$ depending upon the $\mathrm{N}$ source. From 50 to $60 \%$ of this $\mathrm{N}$ was released in the form of amino acids upon acid hydrolysis. With both asparagine and $\mathrm{NaNO}_{3}$ as $\mathrm{N}$ sources the same amino acids were isolated from $6 \mathrm{~N} \mathrm{HCl}$ hydrolysates. Quantitatively, however, there were differences in the percentage distribution of the amino acids.
\end{abstract}

Additional Index Words: soil fungi; anthraquinone formation by fungi; anthraquinones in fungal polymers; anthraquinones released from fungal polymers; phenols released from fungal polymers; amino acids in fungal polymers.

$\mathrm{M}$ CGRATh $(18,19,20)$ extracted several anthraquinones in amounts up to $120 \mathrm{ppm}$ from a variety of Irish and Canadian soils. Chrysotalunin, a hydroxybianthraquinone derived from chrysophanol, was more prominent than monomer anthraquinones such as physcion and chrysophanol. These anthraquinones could be of plant or microbial origin (27). Their occurrence, however, under heath or grass suggests a microbial origin. Numerous soil fungi including Penicillium, Aspergillus, and Trichoderma species synthesize anthraquinones (38) and the possibility that these and other quinones (in addition to simple phenolic compounds) are important constituent units in the forma-

\footnotetext{
Contribution from the Institut für Biochemie des Bodens Forschungsanstalt für Landwirtschaft, Braunschweig, Germany. Centro de Edafologia y Biologia Aplicada del Cuarto. Sevilla, Spain, and the Dept. of Soil Science \& Agric. Eng.. Univ. of California, Riverside CA 92502. The authors give thanks to Prof. Dr. Steglich. Berlin; Prof. Dr. K. Wagner, Munich: and Prof. Dr. M. H. Zenk, Bochum, for furnishing pure anthraquinone samples. They thank Mrs. E. Pleiss for skilled technical laboratory assistance and Prof. Dr. H. Söchtig for his help with the quantative amino acid determinations. $C$. Saiz-Jimenez thanks the Deutsche Forschungsgemeinschaft for a post-doctoral stipend. Received 11 Nov. 1974. Approved 30 Jan. 197.5.

${ }^{2}$ Visiting Scientist at the Inst. of Soil Biochemistry. Biochemist and Professor of Soil Science. Univ. of California. Riverside, respectively.
}

tion of soil humus is receiving more consideration. The socalled P-type humic acids, isolated first by Kumada and Sato $(14,15)$ are associated with perylene quinones, probably of fungal origin (16). Structures related to perylene quinone were also isolated as intermediates in the formation of the dark colored pigment of Daldinia and Aspergillus spp. and according to Bu Lock (6), they are formed by condensation of 1,8-dinaphthol units.

According to Mathur (24) 2-methyl-1,4-naphthoquinone was obtained in amounts up to $10 \%$ from a podzol fulvic acid upon degradation with cultures of Poria subacida. These results were confirmed with cell-free preparations. Evidence for the presence of substantial amounts of quinones in soil humic material has also been obtained by chemical analyses of functional groups and by infrared spectroscopy $(24,25,26,32)$. It is possible that the condensed hydrocarbons isolated either directly from the soil $(5,39)$ or upon zinc dust distillation of humic acids $(8$. $11,13)$ may originate by reduction of more highly condensed quinoid or phenolic structures.

The fringelites of sea sediments discussed by Blumer (4) and by Albrecht and Durisson (1) are dimeric anthraquinones with tetra-hydroxy-naphthodianthrone structures. They are derived from fossil crinoids and undergo a series of hydrations and dehydrations.

For the present paper a study was made of anthraquinones and phenols formed by Eurotium echinulatum, a soil fungus isolated from a Vertizol in southern Spain. The linkage of these compounds into dark-colored pigments similar to humic acid by either phenolase or autoxidative polymerization processes in the culture medium and in the cells during and after termination of growth was followed.

\section{METHODS}

The methods for cultivation and some properties of the darkcolored pigment have been described $(22,23)$. The fungus was cultured in Czapek-Dox medium ( $20 \%$ glucose and $0.2 \%$ $\mathrm{NaNO}_{3}$ ) or in glucose-asparagine medium ( $3 \%$ glucose and $0.5 \%$ asparagine) as described by Martin et al. (21). Growth and polymer formation was much better in the glucose-asparagine medium, but the Czapek-Dox was preferred for monitoring the formation and transformation of phenols and anthraquinones. The phenols and anthraquinones were isolated from the culture media by acidification to $\mathrm{pH} 1.0$ and extraction with peroxide-free ether. Concentrated ether extracts were spotted on thin layer silica gel (KGF 254, Fa, Merck, Darmstadt) plates and chromatographed in two directions $(9,10,12)$ with $\mathrm{CHCl}_{3}-\mathrm{CH}_{3} \mathrm{COOH}(8: 2)$ and dibutylether- $\mathrm{CH}_{3} \mathrm{COOH}(10: 1)$ or by the method of Leistner (17) with $\mathrm{CHCl}_{3}$-ethylacetate- $\mathrm{HCOOH}$ $(5: 4: 1)$ or benzene-ethylformate- $\mathrm{HCOOH}(75: 24: 1)$ in the first and with dibutylether- $\mathrm{CH}_{3} \mathrm{COOH}(10: 1)$ in the second direction. The first developing system gave a better separation of the phenols and the second a better separation of the anthraquinones.

Phenol spots were observed under UV-light at $254 \mathrm{~nm}$ and after specific color reactions with diazotized nitroaniline or sulfanilic acid. Anthraquinones were detected with UV-light at 366 nm and by spraying with $24 \% \mathrm{NaOH}$ (2) or with $0.5 \% \mathrm{Mg}$ acetate (33). To obtain a better separation of the anthraqui- 
nones from the phenols, the ether extracts were added to a silica gel column $(2.5$ by $30 \mathrm{~cm}$ filled with silica gel, Merck, 30-70 mesh) and eluted with benzene-ethylformate-HCOOH (75:21:1). The colored bands were collected and chromatographed in two directions on thin layer plates as described. Samples of known anthraquinones and phenols were cochromatographed with the unknown compounds. Agreement of Rf-values, color reaction, and spectra in the UV and visible light range of extracted spots with the pure anthraquinones or phenols were used as criteria for identification.

The dark-colored humic acid-type pigments were isolated after 3 months from the $\mathrm{NaNO}_{3}$ medium and after 4 to 6 weeks from the asparagine medium. The pads were removed and the media filtered and centrifuged at $15,000 \times \mathrm{g}$. The clear solutions were acidified to $\mathrm{pH} 1$ and the precipitate collected by centrifugation. The precipitate was dissolved in $0.1 \mathrm{~N} \mathrm{NaOH}$ and the solution centrifuged at $15,000 \times g$. The clear solution was dialyzed against distilled water, and the humic polymer recovered by precipitation at pH 1.0, washing, and lyophilization.

The humic acid-type pigments were degraded by the $\mathrm{Na}$ amaigam method $(7,21,22,30,31)$. For this purpose 25 - to $50-\mathrm{mg}$ portions of the polymers were treated with $40 \mathrm{~g}$ of $3 \%$ or $25 \mathrm{~g}$ of $5 \% \mathrm{Na}$-amalgam under $\mathrm{N}_{2}$. Anthraquinones were specifically released by refluxing $50-\mathrm{mg}$ portions under $\mathrm{N}_{2}$ with $50 \mathrm{ml}$ of $1 \%$ Na-dithionite solution for $10 \mathrm{~min}$. The reaction mixtures were acidified and extracted with peroxide-free ether.

For determining the amino acids in the polymers, $200-\mathrm{mg}$ portions of the pigments were hydrolized with $20 \mathrm{ml}$ of $6 \mathrm{~N} \mathrm{HCl}$ for 24 hours at $105 \mathrm{C}$ in sealed tubes under $\mathrm{N}_{2}$. Aliquots of the hydrolysates were spotted on paper and separated in the first direction by high voltage electrophoresis at $\mathrm{pH} 1.9(2.2 \mathrm{kV}$ for $60 \mathrm{~min}$ ) and in the second direction by descending chromatography with sec. butanol-HCOOH- $\mathrm{H}_{2} \mathrm{O}(75: 15: 10)$ as described by Haider and Martin (9). Amino acid spots were noted upon spraying with ninhydrin and their positions compared with known amino acids. For quantitative determination of the amino acids,

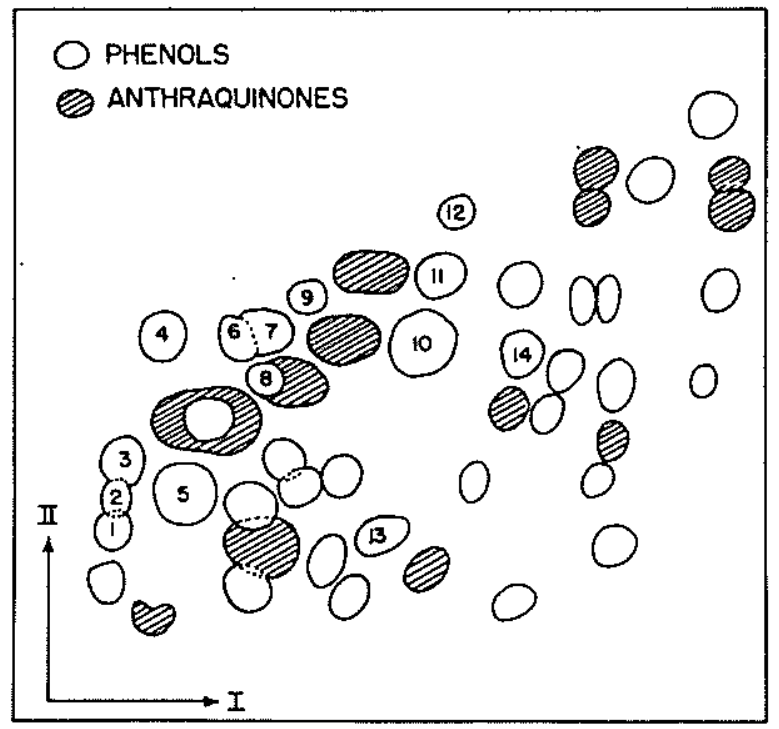

IDENTIFIED PHENOLS
1. 2,4,6-Trihydroxybenzoic acid
2. Gallic acid
3. Phloroglucinol
4. 3,5-Dihydroxybenzoic acid
5. Acetylphloroglucinol
6. Protocatechuic acid
7. Protocatechualdehyde?

8. Catfric acid

9. Orcinol

10. $p$-Hydroxycinnamic acid

11. p-Hydroxybenzoic acid

12. Orsellinic acid

13. 2,3,5-Trihydroxytoluene

14. p.Hydroxybenzaldehyde

Fig. 1-Two dimensional thin layer chromatogram showing phenols and anthraquinones extracted with ether from a 14 day Czapek-Dox culture medium of $E$. echinulatum. The developing solution for the first direction (I) was $\mathrm{CHCl}_{3}-\mathrm{COOH}$ (8:2) and for the second (II) dibutylether- $\mathrm{CH}_{3} \mathrm{COOH}(10: 1)$. the hydrolysates were chromatographed on an amino acid analyzer (Biotronik, Model LC 4010). The amounts of the specific amino acids were calculated on the basis of $\mu$ mole $/ \mathrm{g}$ of the polymer or in percent of the total amino acids recovered plus the ammonia.

\section{RESULTS}

Within 6 to 7 days after inoculation of the culture media with $E$. echinulatum several phenols and anthraquinones could be extracted with ether from the culture solutions. Thin layer chromatography revealed the presence of the anthraquinones, endocrocin, emodin, and physcion, and the phenols, $p$-hydroxycinnamic and $p$-hydroxybenzoic acids. Within 8 to 10 days up to 30 different prominent spots were visible. In addition to those indicated the anthraquinones, dermolutein, questin, questinol, catenarin, erythroglaucin, and dermoglaucin and the phenols orsellinic, protocatechuic, gallic 3,5-dihydroxybenzoic, and 2,4,6-trihydroxybenzoic acids, orcinol, phloroglucinol, acetylphloroglucinol and possibly protecatechualdehyde, $p$-hydroxybenzaldehyde, caffeic acid, and pyrogallol were identified.

After 14 to 18 days about 50 different spots were visible on the plates. In addition to those previously noted the anthraquinones, fallacinol, fallacinal, parietinic acid, and dermocybin and the phenol, 2,3,5-trihydroxytoluene were identified. The distribution of the phenols and anthraquinones on a thin layer chromatographic plate made from the ether extract of a 14-day-old culture of $E$. echinulatum in Czapek-Dox medium may be noted in Fig. 1. Figure 2

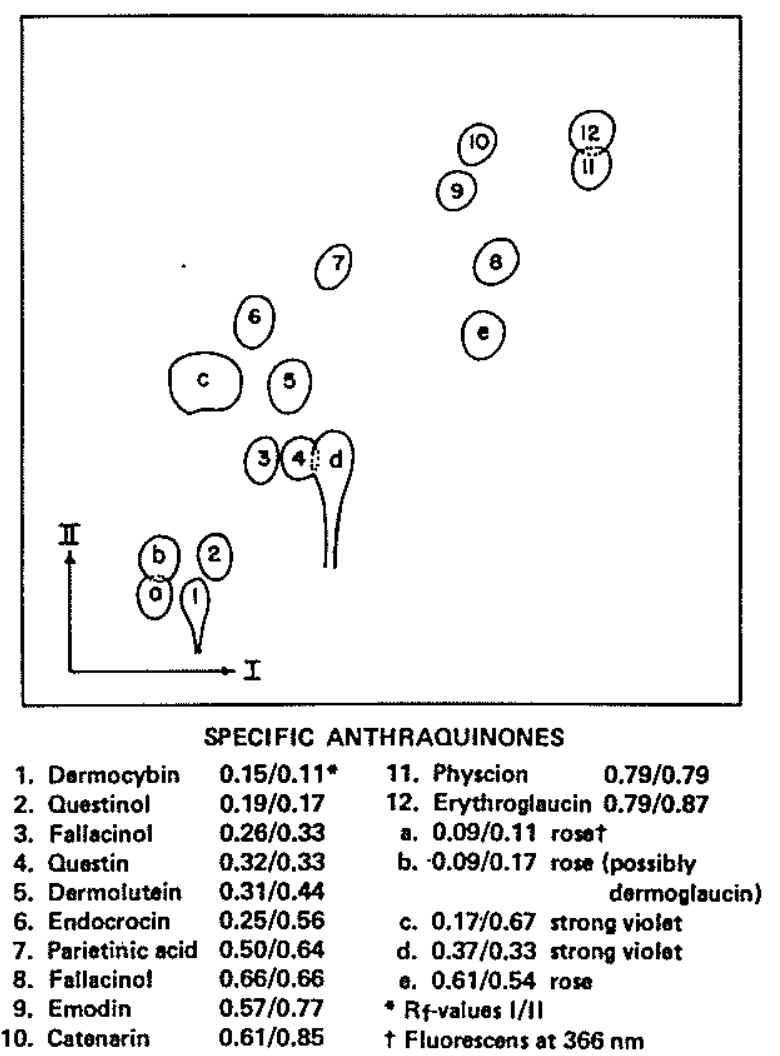

Fig. 2-Two-dimensional thin layer chromatogram showing anthraquinones from 14-day Czapek-Dox culture medium of $E$. echinulatum. The developing solution for the first direction (I) was benzene-ethylformate-HCOOH $(75: 24: 1)$ and for the second (II) dibutylether- $\mathrm{CH}_{3} \mathrm{COOH}$ (10:1). 
shows the distribution of the anthraquinones only after they were separated from a 14-day culture.

The culture media turned from colorless to a reddish yellow within 10 days after inoculation. After 2 to 3 weeks with the asparagine-glucose medium and 1 to 2 months with the Czapek-Dox medium the culture solutions became deep brown to almost black. The pH of the Czapek-Dox medium decreased from an initial pH of 6 to almost 4 and remained there for 4 months. In the glucose-asparagine medium the pH decreased from 6 to 5 in about 10 days and then increased during autolysis and polymer formation to about 7.8. From 0.8 to $1.0 \mathrm{~g} /$ liter of the polymer was recovered from the Czapek-Dox medium and 3.0 to $3.5 \mathrm{~g} /$ liter from the glucose-asparagine medium.

Reductive degradation of the polymers with Na-amalgam yielded about 45 to $70 \%$ ether-soluble products. The higher amounts were released from the low $\mathrm{N}$ polymers obtained from the Czapek-Dox medium. Thin layer chromatography of the ether extracts separated many phenols and anthraquinones (Fig. 3). In addition to anthraquinones, anthrones and anthranols were probably present. These could be formed by the reductive procedure since $\mathrm{Na}$-amalgam degradation of the anthraquinones, emodin, endocrocin, and physcion partially converted the compounds to anthrones, anthranols, and several unidentified substances. Therefore, a conclusive comparison of the spots with known compounds was not possible. Furthermore, a reduction of the anthraquinones to anthracene derivatives may occur. From both pure anthraquinones and the polymer

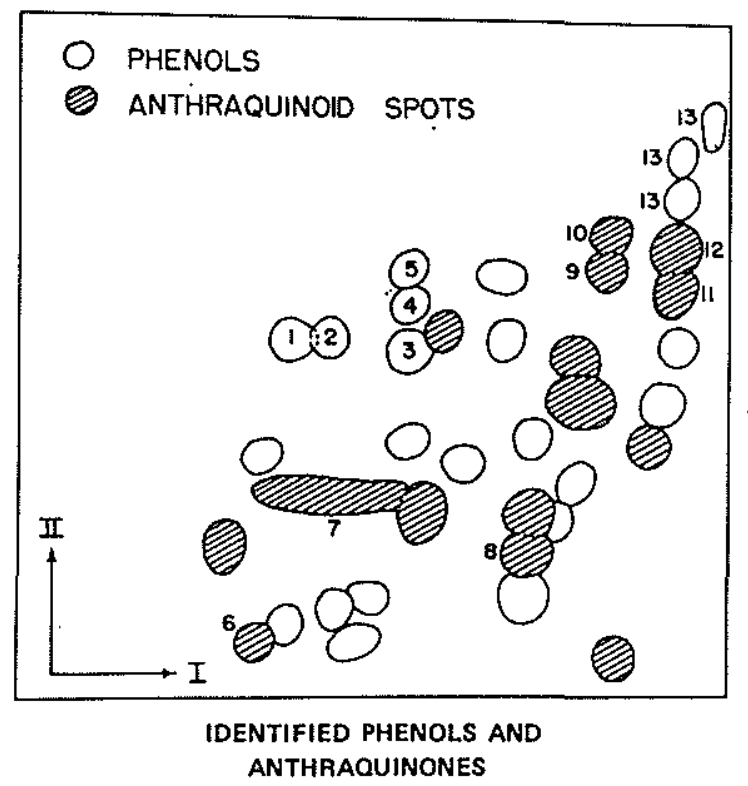
1. Resarcinol
8. Physcion anthrone
2. Orcinol
9. Emodin
3. $p \cdot$ Hydroxycinnamic acid
10. Catenarin
4. $p$-Hydroxybenzoic acid
11. Physcion
12. Erythroglaucin
6. Ouestinol?
7. Endocrocin
13. Possibly anthracane derivatives

Fig. 3-Thin layer chromatogram of phenols and anthraquinoid compounds released during Na-amalgam reductive degradation of the humic acid-type polymers of $E$. echinulatum. The developing sulution for direction (I) was $\mathrm{CHCl}_{3} \mathrm{COOH}$ (8:2) and for (II) dibutylether-CH; $\mathrm{COOH}(10: 1)$. several spots with a pronounced blue fluorescein under UV-light at $254 \mathrm{~nm}$ were observed. These were located near the solvent front. With less polar solvents, e.g., cyclohexane- $\mathrm{CHCl}_{3}(80: 20)$ or with $n$-heptane, these compounds had similar but not identical $R_{f}$-values to anthracene. The spots reacted positively with iodine vapor and with formaldehyde- $\mathrm{H}_{2} \mathrm{SO}_{4}$ reagent (13).

Treatment of the polymers with $\mathrm{Na}$-dithionite solution released largely anthraquinones. No anthrones or anthranols were noted. The distribution of the spots on thin-layer plates upon chromatography with ethylformate-benzene$\mathrm{HCOOH}$ in the first and with dibutylether-acetic acid in the second direction is shown in Fig. 4.

The $\mathrm{N}$ content of the polymer ranged from about $1 \%$ in the Czapek-Dox medium to $4.5 \%$ in the asparagine-glucose medium. About $50 \%$ of the total $\mathrm{N}$ in the polymers was released by acid hydrolysis. Two dimensional separation of the amino acids in the hydrolysate on paper by electrophoresis and descending chromatography yielded arginine, histidine, glycine, alanine, proline, methionine, tyrosine, valine, phenylalanine, leucine and/or isoleucine, and 3 nonidentified amino acids. Hydrolysates from the polymers of both culture media showed the same qualitative distribution of the amino acids. The quantitative amino acid analysis of the hydrolysates is presented in Table 1 . The amounts of amino acids in $\mu$ mole $/ 1 \mathrm{~g}$ of polymer and in percent of the individual amino acids from the total amino acids plus ammonia are given. For comparison this table also shows the amino acids from hydrolysates of Stachybotrys chartarum polymers isolated from a glucose-asparagine culture medium and from the fungus cells, respectively. The amounts of amino acids from the glucose-asparagine medium varied

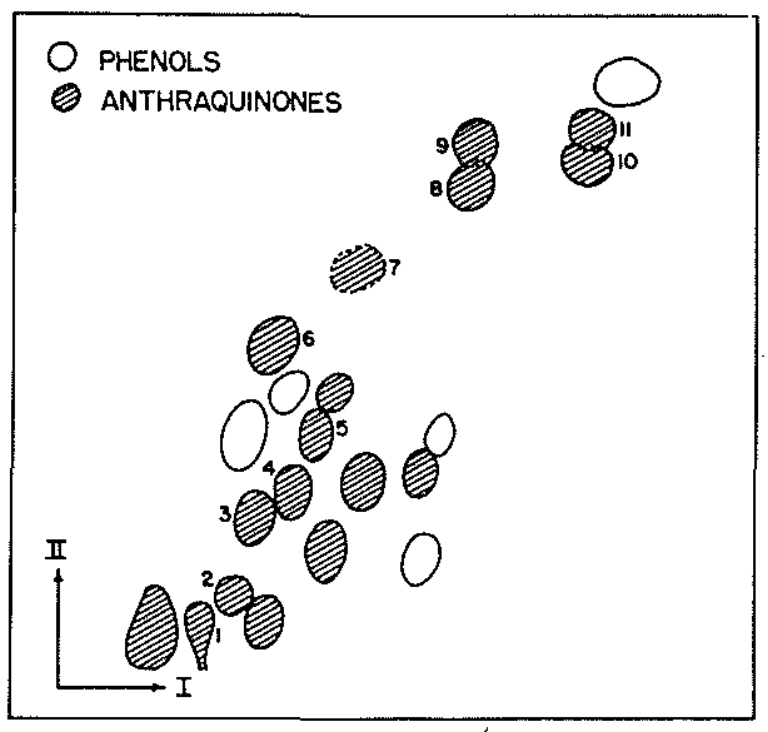

IDENTIFIED COMPOÚNDS
1. Dormocybin
2. Questinol
5. Dormolutein
6. Endocrocin
3. Fallacinol
7. Parietinic acid?
4. Questin?
8. Emodin
9. Catonarin
10. Physcion
11. Erythroglaucin

Fig. 4-Thin layer chromatogram of anthraquinones released from $E$. echinulatum humic acid-type polymer by treatment with Na-dithionite solution. The developing solution for direction (I) was benzene-ethylformate-HCOOH (75:24:1) and for (II) dibutylether- $\mathrm{CH}_{3} \mathrm{COOH}$ (10:1). 
Table 1-Distribution of amino acids in $6 \mathrm{~N} \mathrm{HCl}$ hydrolysates of humic acid-type polymers from $E$. echinulatum and Stachybotrys chartarum

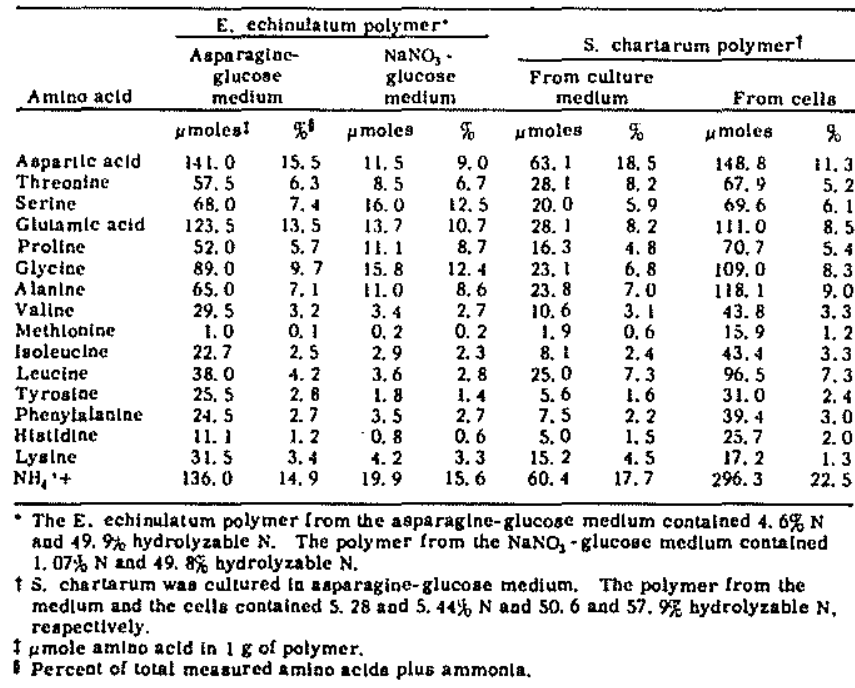

from 1 to $141 \mu$ mole/g and from Czapek-Dox medium 0.2 to $16 \mu \mathrm{mole} / \mathrm{g}$ of the various amino acids were found. Similar quantities were present in the $S$. chartarum polymers.

\section{DISCUSSION}

Eurotium echinulatum cultured on glucose as a carbon source synthesized numerous phenols and anthraquinones. The phenols, $p$-hydroxycinnamic, $p$-hydroxybenzoic, and orsellinic acids and acetyl-phloroglucinol are formed either through the shikimate or the acetate-malonate pathway (3). These are transformed into other phenols by degradation of the $\mathrm{C}_{3}$-side chain, decarboxylation, oxidation of the methyl to carboxyl groups and by introduction of additional $\mathrm{OH}$-groups into the ring. These transformations follow similar sequences as noted for Epicoccum nigrum (9) and Stachybotrys chartarum (22).

- The anthraquinones, endocrocin and emodin, are also formed through the polyketide pathway from nonaromatic precursors (29). Emodin could either originate from endocrocin by decarboxylation or by a separate biosynthesis directly from polyacetates $(36,37)$. A possible sequence of the transformations of emodin and endocrocin into other anthraquinones found in the $E$. echinulatum culture media is given in Fig. 5. According to Steglich (36) dermolutein would be derived from endocrocin; however, as indicated by Steglich et al. (35) the other anthraquinones methylated in the 8-position, questin and questinol, are probably also derived from endocrocin. Physcion is formed by methylation of emodin in position 6 . This compound can be hydroxylated to form erythroglaucin, dermoglaucin, and dermocybin. Stepwise oxidation of the 3-methyl group of physcion, forms fallacinol, fallacinal, and parietinic acid. During the period of rapid polymer formation the phenols and anthraquinones largely disappeared from the medium. At the termination of incubation only small amounts of physcion, fallacinol, and fallacinal (and possibly questin and questinol) were still present in free forms and the simple phenols had largely disappeared. It is highly probable that these phenols and anthraquinones were linked into the

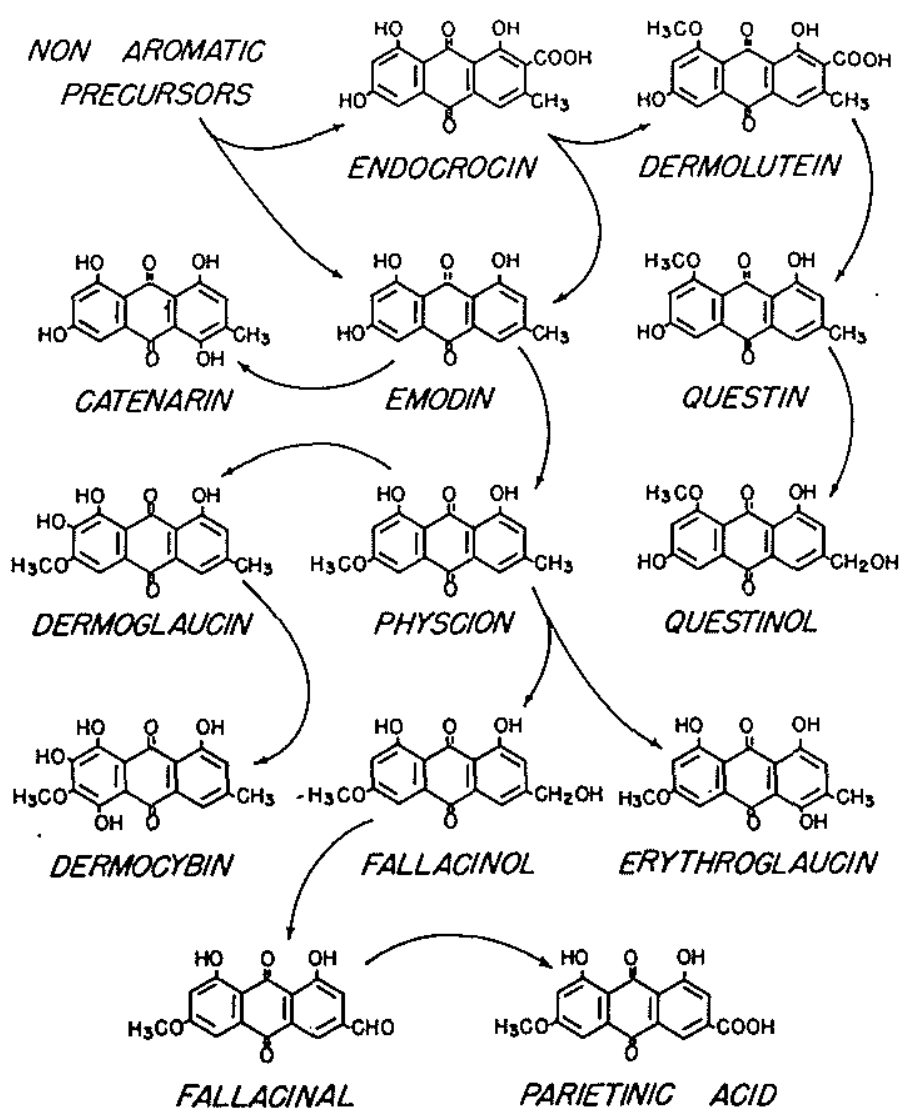

Fig. 5-Possible transformations in the formation of anthraquinones by $E$. echinulatum.

developing polymers. No anthraquinones or phenols could be extracted with ether or alcohol from the polymers even after exhaustive methylation with diazomethane. Reduction of the polymers with Na-amalgam yielded phenols, anthraquinones, anthrones, anthranols, and probably anthracene derivatives. The anthrones, anthranols and the anthracenes, however, appear to be formed by the reductive degradation treatment and are not present as such in the polymers as supported by the release of anthraquinones only upon $\mathrm{Na}$ dithionite treatment. According to Shibata et al. (34) and Ogihara et al. (36) the reductive cleavage with dithionite splits the latter into the single anthraquinones. Piper and Posner (30) using dimer model phenol compounds found that the Na-amalgam reduction reaction splits either linkages with the release of free phenols.

In addition to phenols and anthraquinones, amino acid compounds were important constituents of the pigments as indicated by the release of amino acids upon acid hydrolysis. They represented from 20 to $50 \%$ of the hydrolyzable $\mathrm{N}$. The $6 \mathrm{~N} \mathrm{HCl}$ hydrolysis solubilizes more organic material and $\mathrm{N}$ than can be accounted for as free amino acids and ammonia. It is possible that some fragmentation of the molecules takes place and much of the $\mathrm{N}$ not present as free amino groups or ammonia could be in the form of amino acid compounds linked to phenyl rings through the amino group. This linkage is resistant to $6 \mathrm{~N} \mathrm{HCl}$ hydrolysis $(30,31)$.

The $\mathrm{N}$ source used in the culture media influenced the content of the polymers, namely, $1 \% \mathrm{~N}$ with $\mathrm{NaNO}_{3}$ and $4.5 \%-\mathrm{N}$ with-asparagine. About $50 \%$ of the $\mathrm{N}$ was teleased 
upon $6 \mathrm{~N} \mathrm{HCl}$ hydrolysis of both the high and low $\mathrm{N}$ polymers and the same amino acids were released upon hydrolysis of both. Quantitatively, the amounts of amino acids per gram of the polymers were much higher in the polymer from the asparagine than from the $\mathrm{NaNO}_{3}$ medium. When the individual amino acids were calculated in percent of the total measured plus the ammonia some amino acids such as threonine, valine, isoleucine, phenylalanine, and lysine showed similar values while others such as aspartic acid, serine, proline, glycine, and tyrosine were different. This indicates that the $\mathrm{N}$ source exerts an important influence on the amino acid composition of the fungal polymers. Therefore, definite conclusions as recently published by Ortiz de Serra et al. (28) based on single fungal polymer preparations prepared with one $N$ source (asparagine) cannot properly be made since the amount and the composition of the hydrolyzable amino acid fraction of fungal humic acids vary greatly and are dependent upon the culture conditions during their formation. Also the fungus species is important as indicated by a different percentage composition of the hydrolyzable amino acid fraction from $S$. chartarum polymers.

The synthesis of humic acid-like polymers by a soil fungus with phenols and anthraquinones as constituent units suggests some new aspects of soil humus formation. Further experiments are needed to explain the mechanism of polymer formation and the significance of these pigments with respect to soil humus formation under natural conditions.

\section{LITERATURE CITED}

1. Albrecht, P., and G. Durisson. Biogene substanzen in sedimenten und fossilien. Angew. Chemie 83:221-238.

2. Ashley, J. N., H. Raistrick, and T. Richards. 1939. The crystalline colouring matters of species in Aspergillus glatucus series. Biochem. J. 33:1291-1303.

3. Bentley, R., and I. M. Campbell. 1968. Secondary metabolism of fungi. 483. p. 415-483. In M. Florkin and E. H. Stotz (ed.) Comprehensive biochemistry, Vol. 20. Elsevier Publishing Co., Amsterdam.

4. Blumer, M. 1960. Pigments of a fossil echinoderm. Nature (London) 188:1100-1101.

5. Blumer, M. 1961. Benzpyrene in soils. Science 134:474 475 .

6. Bu Lock, J. D. 1967. Essays in biosynthesis and microbial development. J. Wiley \& Sons, New York. 71 p.

7. Burges, W. A., H. M. Hurst, and B. Walkden. 1964. The phenolic constituents of humic acid and their relation to the lignin of the plant cover. Geochim. Cosmochim. Acta 28:1547-1554.

8. Cheshire, M. V., P. A. Cranwell, and R. D. Haworth. 1968. Humic acids. Tetrahedron 24:5155-5167.

-9. Haider, K., and J. P. Martin. 1967. Synthesis and transformation of phenolic compounds by Epicoccum nigrum in relation to humic acid formation. Soil Sci. Soc. Am. Proc. 31:766-772.

10. Haider. K., and J. P. Martin. 1970. Humic acid-type phenolic polymers from Aspergillus sydowi culture medium, Stachyborrys spp. cells and autoxidized phenol mixtures. Soil Biol. Biochem. 2:145-156.

11. Hansen. E., and M. Schnitzer. 1967. Zn-dust distillation and fusion of a soil humic and fulvic acid. Soil Sci. Soc. Am. Proc. 33:29-36

12. Kucharczyk, M., J. Fohl, and J. Vymětal. 1963. Dünnschicht-chromatographie von aromatischen Kohienwasserstoffen und einigen heterocyclischen Verbindungen. J. Chromasog. $11: 55-61$

13. Kunadi, K., and H. M. Hurst. 1967. Green humic acid and its possible origin as a fungal metabolite. Nature (London) 214:631.

14. Kumada, K., and Y. Matsue. 1970. Studies on the composition of aromatic nuclei of humus. Part I. Determination of some aromatic nuclei of humic acid. Soil Sci. Plant Nutr. (Tokyo) 16:250-255.

15. Kumada, K., and O. Sato. 1962. Chromatographic separation of green humic acid from podzol humus. Soil Sci. Plant Nutr. (Tokyo) 8:31-33.

16. Kumada, K., and O. Sato. 1967. Studies on the chemical properties of P-type humic acid, p. 131-133. In B. Novák and V. Rypácek (ed.), Prague, Symp. humus et planta IV.

17. Leistner, E. 1971. A second pathway leading to anthraquinones in higher plants. Phytochemistry 10:3015-3020.

18. McGrath, D. 1967. Nature and distribution in Irish soils of a new pigment. Nature 215:1414.

19. McGrath, D. 1970. Chrysotalunin, a new bianthraquinone from soil. Chem. Ind. (Lond.) 42:1353.

20. McGrath, D. 1972. Hydroxyanthraquinones in soil. Geoderma 7:167-176.

21. Martin, J. P., and K. Haider. 1969. Phenolic polymers of Stachybotrys atra, Stachybotrys chartarum and Epicoccum nigrum in relation to soil humus formation. Soil Sci. 107: 260-270.

22. Martin, J. P., K. Haider, and C. Saiz-Jimenez. 1974. Sodium amalgam reductive degradation of fungal and model phenolic polymers, soil humic acids and simple phenolic compounds. Soil Sci. Soc. Am. Proc. 38:760-764.

23. Martin, J. P., S. J. Richards, and K. Haider. 1967. Properties and decomposition and binding action in soil of a "humic acid" synthesized by Epicoccum nigrum. Soil Sci. Soc. Am. Proc. 31:657-662.

24. Mathur, S. P. 1971. Characterization of soil humus through enzymatic degradation. Soil Sci. 111:147-157.

25. Mathur, S. P. 1972. Evaluation of a reductometric titration method for determining quinones in soil humus. Soil Sci. Soc. Am. Proc. 36:175-176.

26. Mathur, S. P. 1972. Sepctroscopic and chemical evidence of quinones in soil humus. Soil Sci. 113:136-139.

27. Ogihara, Y., W. Kobayashi, and S. Shibata. 1968. Bianthraquinones of Penicillium islandicum. Tetrahedron Lett. $1881-1886$

28. Ortiz de Serra, M. I., F. J. Sowden, and M. Schnitzer. 1973. Distribution of nitrogen in fungal "humic acids." Can. J. Soil Sci. 53:125-127.

29. Packter, N. M. 1973. Biosynthesis of acetate-derived compounds. J. Wiley \& Sons, London. 203 p.

30. Piper, T. J., and A. M. Posner. 1972a. Sodium amalgam reduction of humic acid. II. Application of the method. Soil Biol. Biochem. 4:525-531.

31. Piper, T. J., and A. M. Posner. 1972b. Sodium amalgam reduction of humic acid. I. Evaluation of the method. Soil Biol. Biochem. 4:513-523.

32. Schnitzer, M., and R. Riffaldi. 1972. The determination of quinone groups in humic substances. Soil Sci. Soc. Am. Proc. 36:772-777.

33. Shibata, S., M. Takito, and O. Tamaka. 1950. Paper chromatography of anthraquinone pigments. J. Am. Chem. Soc. 72:2789-2790.

34. Shibata, S., O. Tanaka, and I. Kitagawa. 1955. Metabolic products of fungi. V. The structure of skyrin. Phar. Bull. (Tokyo) 3:278-283.

35. Steglich, W. 1972. The biosynthesis of fungal quinones Hoppe-Seyler Z. f. Physiol. Chemie 353:124-125.

36. Steglich, W., R. Arnold, W. Lösel, and W. Reisinger. 1972. Biosynthesis of anthraquinone pigments in Dermocybe. J. Chem. Soc. (Chemical Commun.), 102-103.

37. Steglich, W., W. Lösel, and V. Austel. 1969. Anthrachinonpigmente aus Dermocybe sanguinea (Wulf, ex $\mathrm{Fr}$.) Wünsche und $D$. semisanguinea (Fr.). Chem. Ber. 102 4104-4118.

38. Thomson, R. H. 1971. Naturally occurring quinones. 2nd Ed. Academic Press. London and New York. 734 p.

39. Wagner, K. H., and I, Siddiqui. 1973. Gefährliche Stoffe in Bodenverbesserungsmitteln. Naturwissenschaft 60:160-161. 